\title{
Variabilidad estadística de cargas vivas en edificios
}

Jorge Fernando Márquez Peñaranda ${ }^{1}$

\section{Resumen}

Este trabajo presenta una importante aplicación de la estadística en la ingeniería civil relacionada con el análisis probabilístico de cargas vivas en edificios el cual toma en cuenta la variabilidad espacial y temporal de las mismas y muestra curvas de carga para análisis y diseño simplificadas obtenidas a partir de mediciones reales efectuadas en la ciudad de San José de Cúcuta.

Palabras clave: Carga viva, área de influencia, probabilidad de excedencia.

\section{Abstract}

Este trabajo presenta una importante aplicación de la estadística en la ingeniería civil relacionada con el análisis probabilístico de cargas vivas en edificios el cual toma en cuenta la variabilidad espacial y temporal de las mismas y muestra curvas de carga para análisis y diseño simplificadas obtenidas a partir de mediciones reales efectuadas en la ciudad de San José de Cúcuta.

Key words: Live load, area of influence, likely to leave.

\section{Introducción}

L a carga viva puede definirse como la carga gravitacional actuante sobre una componente estructural que varía de forma importante en magnitud y posición en el transcurso del tiempo. Puede decirse que ésta se produce básicamente por la suma de una porción sostenida y una extraordinaria [4]. La primera toma en cuenta el aporte del uso normal (por ejemplo peso de mobiliario y personas que ocupan habitualmente el lugar) y varía muy poco en frecuencia y magnitud durante el tiempo de ocupación de un mismo usuario. La porción extraordinaria suele producirse por sucesos poco comunes tales como concentración de pesos de personas o muebles en el caso de una remodelación del lugar. Estas componentes de carga se modelan buscando su ocurrencia más desfavorable para el elemento estructural afectado en el tiempo de vida de la estructura considerando su aleatoriedad espacial y temporal. Como resultado se proponen curvas simplificadas que pueden ser comparadas con las propuestas por la práctica y normas asociadas al análisis y diseño estructural de edificios, adaptadas para el caso particular de edificios de oficinas.

\footnotetext{
${ }^{1}$ Ingeniero Civil, Magíster en Ingeniería Sismo Resistente, Especialista en Ingeniería Sismo Resistente, Especialista en Gestión y Desarrollo de Proyectos de Investigación. Profesor Asociado Universidad Francisco de Paula Santander. Grupo de Investigación "Construcciones Civiles Armónicas".
} 


\section{DESARROLLO DEL MODELO}

Para conocer de forma real las características de las cargas vivas en un área determinada es necesario medir su magnitud y posición. Sin embargo esta medida se hace en un instante dado y debe proveerse información que involucre la variabilidad en el tiempo de ella. Además los datos obtenidos con este fin usualmente son estimaciones gruesas de pesos totales de elementos o personas, la posición de su centro de gravedad y la medida del área que afectan.

Para modelar la intensidad de carga sostenida $\mathrm{w}(\mathrm{x}, \mathrm{y})$ se usa un modelo probabilístico lineal [2], [4] donde m es la media de toda la población de cargas, $g_{e}$ y $g_{p}$ y e $(x, y)$ son variables aleatorias con media cero usadas para ajustar las variaciones de intensidad entre edificios, entre pisos de un mismo edificio y sobre un área tributaria de un mismo piso respectivamente (ver figura 1).

$$
w(x, y)=m+\gamma_{e}+\gamma_{p}+\varepsilon(x, y)
$$

FIGURA 1. Componentes de la intensidad de carga

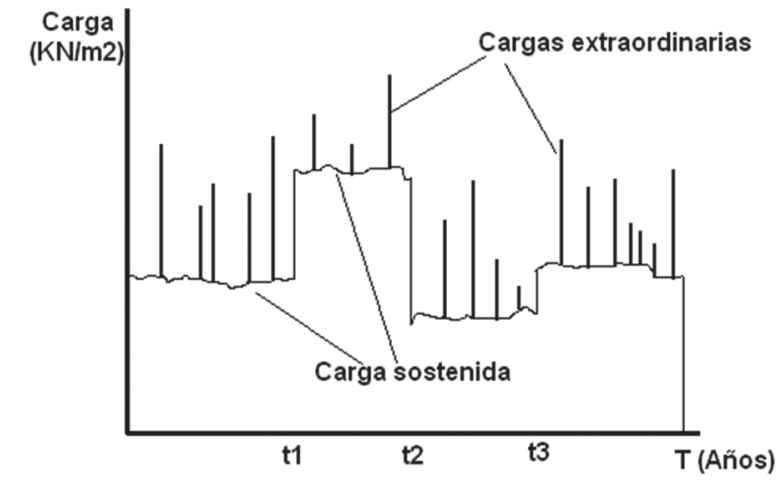

La variable $\mathrm{e}(\mathrm{x}, \mathrm{y})$ describe el proceso estocástico de carga e inicialmente se supone que corresponde a un proceso correlacionado tal que:

$$
\operatorname{cov}\left[\varepsilon\left(x_{o}, y_{o}\right) \varepsilon\left(x_{1}, y_{1}\right)\right] \neq 0
$$

La varianza de $\mathrm{w}(\mathrm{x}, \mathrm{y})$ se calcula como la suma de las varianzas de las variables aleatorias involucradas es decir:

$$
\operatorname{var}(w(x, y))=\sigma_{e}^{2}+\sigma_{p}^{2}+\sigma_{s p}^{2}
$$

La carga unitaria observada en las mediciones de campo U(A) debe alimentar apropiadamente el modelo. Para ello se plantea la su relación con $\mathrm{w}(\mathrm{x}, \mathrm{y})$, su valor esperado y su varianza así [2], [4]:

$U(A)=\frac{1}{A} \int_{0}^{a} \int_{0}^{b} w(x, y) d x d y$

$E\left(U(A)=\frac{\int_{0}^{a} \int_{0}^{b} E(w(x, y) d x d y}{A}=\frac{m \int_{0}^{a} \int_{0}^{b} d x d y}{A}=m\right.$

$\operatorname{var}(U(A))=\frac{1}{A^{2}} \int_{0}^{a} \int_{0}^{a} \int_{0}^{b} \int_{0}^{b} \operatorname{cov}\left[w\left(x_{0}, y_{0}\right), w\left(x_{1}, y_{1}\right)\right] d x_{0} d x_{1} d y_{0} d y$

Como la única variable de $w(x, y)$ es e(x,y) su covarianza puede expresarse como:

$\operatorname{cov}\left[w\left(x_{0}, y_{0}\right) w\left(x_{1}, y_{1}\right)\right]=\sigma_{e}^{2}+\sigma_{p}^{2}+\operatorname{cov}\left[\varepsilon\left(x_{0}, y_{0}\right) \varepsilon\left(x_{1}, y_{1}\right)\right]$

En este punto es apropiado el uso de una forma de decaimiento exponencial para el último término de la ecuación anterior con lo que la misma se reescribe como:

$\operatorname{var}(U(A))=\frac{1}{A^{2}} \int_{0}^{a} \int_{0}^{a} \int_{0}^{b} b_{0}^{b}\left[\sigma_{e}^{2}+\sigma_{p}^{2}+r_{c}^{*} \exp \left(-\frac{r^{2}}{d}\right)\right] d x_{0} d x_{1} d y_{0} d y_{1}$

donde $\boldsymbol{d}$ es una constante de decaimiento, $\boldsymbol{r}$ es la distancia horizontal entre dos puntos cualesquiera y $\boldsymbol{r}_{c}$ el coeficiente de correlación entre los valores de carga de los puntos $\mathrm{A}\left(\mathrm{x}_{0}, \mathrm{y}_{0}\right)$ y $\mathrm{B}\left(\mathrm{x}_{1}, \mathrm{y}_{1}\right)$. En este caso como los puntos $\mathrm{A}$ y $\mathrm{B}$ están en un mismo piso $r_{c}=1$. Usando integrales iteradas e introduciendo la función error $\mathrm{f}(\mathrm{z})$ se llega a:

$$
\begin{gathered}
\operatorname{var}(U(A))=\sigma_{e}^{2}+\sigma_{p}^{2}+\sigma_{u}^{2} * \frac{K(A)}{A}=\sigma_{s}^{2}=e+\frac{f}{A} \\
\sigma_{u}^{2}=\pi^{*} d^{*} \sigma_{s p}^{2}
\end{gathered}
$$

$k(A)=\left[\operatorname{fer}\left(\sqrt{\frac{A}{d}}\right)-\sqrt{\frac{d}{A \pi}} *\left(1-\exp \left(-\frac{A}{d}\right)\right)\right]^{2}$

Cuando se analizan puntos de diferentes pisos es necesario introducir el valor de $\boldsymbol{r}_{c}$ calculado según los datos de los pisos estudiados en la ecuación (8). En este caso los valores de carga unitaria U(An) cumplirán las siguientes relaciones: 


$$
U\left(A_{n}\right)=\frac{\sum_{1}^{n} \int_{0}^{a} \int_{0}^{b} w(x, y) d x d y}{n^{*} A}
$$

$\operatorname{var}\left(U\left(A_{n}\right)\right)=\sigma_{e}^{2}+\frac{\sigma_{p}^{2}}{n}+\frac{\sigma_{u}^{2} * K(A)}{n * A}+r_{c} * \frac{\sigma_{s p}^{2} * K(A)}{n * A} *(n-1)$

Para definir las cargas más desfavorables según el efecto se usa el concepto de superficies de influencia (ver figura 2). Si $I(x, y)$ describe la superficie de influencia de un efecto dado (flexión, carga axial, cortante, torsión) el valor total del mismo $\mathrm{F}(\mathrm{A})$ producido en un área $\mathrm{A}=\mathrm{a}^{*} b$ puede calcularse como:

$$
F(A)=\int_{0}^{a} \int_{0}^{b} w(x, y) * I(x, y) d x d y
$$

FIGURA 2. Ejemplo de superficie de influencia para carga de columna central [5].

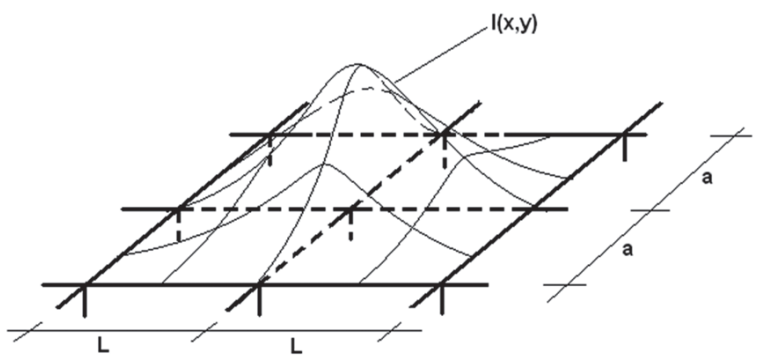

Normalizando los valores de $I(x, y)$ haciendo $\mathrm{x}=\mathrm{x} / \mathrm{Lx}$ y $\mathrm{y}=\mathrm{y} / \mathrm{Ly}$ el valor esperado y la varianza para cada efecto se pueden calcular como:

$$
\begin{gathered}
E(F(A))=L y * L x \int_{0}^{1} \int_{0}^{1} E(w(x, y)) * I(x, y) d x d y=m^{*} A^{*} m_{I} \\
\operatorname{var}\left(F(A)=\left(\sigma_{e}^{2}+\sigma_{p}^{2}\right) * A^{*} m_{I}^{2}+f^{*} A \int_{0}^{1} \int_{0}^{1} I^{2}(x, y) d x d y\right. \text { (12) } \\
m_{I}=\frac{\int_{0}^{a} \int_{0}^{b} I(x, y) d x d y}{A} \\
V_{I}=m_{I} * A
\end{gathered}
$$

Los parámetros asociados a una carga equivalente uniformemente distribuida $\mathrm{Cu}(\mathrm{A})$ que produzca el máximo efecto estudiado pueden ser calculados dividiendo la primera parte de las ecuaciones (12) sobre el valor $V_{I}$ y la segunda parte por el valor $V_{I}^{2}$ con lo que se obtiene:

$$
\begin{gathered}
E(C u(A))=m \\
\operatorname{var}(C u(A))=\sigma_{e}^{2}+\sigma_{p}^{2}+\frac{f}{(13))^{l}}\left[\frac{\int_{0}^{1} \int_{0}^{1} I^{2}(x, y) d x d y}{m_{I}{ }^{2}}\right] \\
\operatorname{var}(C u(A))=e+K e * \frac{f}{A}
\end{gathered}
$$

Para estudiar la variabilidad de la carga sostenida en el tiempo y el espacio se usan ahora los datos medidos para cargas unitarias U(A) tomando su media y varianza como buena estimación de esos parámetros para la población siempre que así lo indique un bajo coeficiente de variación. Además al usar frecuencias relativas acumuladas para cargas y efectos según cada área se verifica la bondad del ajuste a una distribución Gamma usando el estadígrafo de Kolmogorov-Smirnov para grados de significancia del $1 \%$ y $5 \%$. La función distribución de probabilidad Gamma tiene la forma [2]:

$$
\begin{aligned}
& f(x)=\frac{\lambda(\lambda x)^{k-1} * \exp (-\lambda x)}{\Gamma(x)}, \text { con } x \geq 0 \\
& k=\frac{E(x)^{2}}{\operatorname{var}(x)} \quad y \quad \lambda=\frac{E(x)}{\operatorname{var}(x)}(14)
\end{aligned}
$$

donde $\mathrm{x}$ es la variable de interés y $\mathrm{f}(\mathrm{x})$ es la función gamma.

Como un área está sometida a diversos cambios de usuario durante el tiempo total de uso de la edificación es necesario investigar además el comportamiento de las cargas máximas en función del tiempo. Para lo anterior se asume que el proceso aleatorio de carga es homogéneo en el tiempo y el espacio, es decir se acepta que las cargas sostenidas sucesivas en el tiempo sobre una misma área son independientes y tendrán la misma distribución de probabilidad que las cargas sostenidas en un punto arbitrario del tiempo. Por lo anterior se adopta la misma distribución Gamma para describir la variabilidad de las cargas en el tiempo.

Para evaluar los máximos valores de carga sostenida $\mathrm{M}$ de un mismo piso en el tiempo durante todo el tiempo de vida útil del edificio $\mathrm{T}$ se usa la distribución acumulativa $\operatorname{FDA}_{\mathrm{M}}(\mathrm{a})$ : 
Donde $\mathrm{a}_{\mathrm{s}}$ es la carga sostenida asociada a un percentil dado y $\mathrm{Cu}(\mathrm{t})$ representa la carga sostenida equivalente uniformemente distribuida en función del tiempo.

Usando las reglas de propiedad condicional para eventos independientes se determina el primer factor de la ecuación anterior mediante integración de la función $\mathrm{f}(\mathrm{x})$ dada en la ecuación (14). Para evaluar el segundo factor se acepta que los cambios de usuario siguen un proceso de Poisson, luego:

$$
\begin{gathered}
P\left[C u(t) \leq \alpha_{S} \text { para } 0 \leq t \leq T\right]=\exp \left(-v_{\alpha} \Delta t\right) \\
v_{\alpha} \Delta t=P\left[C u(t)>\alpha_{s} \text { en } \Delta t\right]
\end{gathered}
$$

siendo $\mathbf{n}_{\mathbf{a}}$ la rata media en que $\mathrm{Cu}(\mathrm{t})>\mathbf{a}_{\mathbf{s}}$. Con lo anterior y definiendo $\mathbf{n}_{\mathrm{s}}$ como la rata promedio anual de cambios de usuario puede reescribirse la $\mathrm{FDA}_{\mathrm{M}}(\mathrm{a})$ como:

$$
F D A_{M}\left(\alpha_{s}\right)=\exp \left[-v_{s} * T\left(1-F D A s\left(\alpha_{s}\right)\right]\right.
$$

En este momento es posible definir las cargas $\mathbf{a}_{\mathrm{s}}$ que tengan una probabilidad muy pequeña de ser excedidas eligiendo percentiles altos de $\operatorname{FDA}_{\mathrm{M}}\left(\mathrm{a}_{\mathrm{s}}\right)$ y con ello seleccionar parcialmente el nivel de confiabilidad que tendrá la edificación.

Una buena aproximación al aporte de cargas extraordinarias puede lograrse simulando agrupamientos de muebles y personas por separado en áreas específicas [5]. Se ha encontrado que las cargas producidas por aglomeración de personas generan mayores valores de efectos [4]. Si en una celda de área Ac existen $\mathrm{Ni}$ cargas $\mathrm{Pj}$ y el total de todas éstas en un área $A$ es de $n$ cargas $P j$ las siguientes expresiones son válidas (ver figura 3 ):

$$
\begin{aligned}
& M_{P}=\frac{\sum_{1}^{n} P i}{n}, \quad n=\sum_{1}^{L} N i \quad, \quad M_{N}=\frac{n}{L} \\
& M_{E}=\frac{\sum_{1}^{n} P i}{A}=\frac{M_{P} * n}{A}=\frac{M_{P} * \sum_{1}^{L} N i}{A}=\frac{M_{P} * M_{N} * L}{A} \\
& \sigma_{E}{ }^{2}=\frac{L *\left(M p^{2} * M_{N}{ }^{2}+M_{N}{ }^{2} *{\sigma_{P}}^{2}+M_{P}{ }^{2} * \sigma_{P}{ }^{2}\right) * K e}{A^{2}}
\end{aligned}
$$


asciende a unas 700 oficinas distribuidas en 30 edificios. Con el fin de investigar todos los efectos incluido el producido por la cantidad de niveles en cargas de columnas la muestra se condicionó al incluir solo edificios de más de tres pisos que tuviesen información geométrica cierta en planos. Usando muestreo por conglomerados se estudiaron solo cuatro edificios con alturas entre diez y doce pisos.

Las variables de entrada más importantes usadas en este trabajo se refieren a carga viva sostenida, tiempo de cambio de la misma y magnitud y tiempo de ocurrencia de eventos de carga extraordinaria.

Para la captura de información se diseñaron formatos y encuestas, se revisaron planos y se estudiaron pesos típicos de mobiliario y personas. Los datos de campo están asociados a cargas sostenidas concentradas Ps, áreas de celdas Ac y tiempos de cambios de usuarios.

Los percentiles usados para los máximos de carga sostenida se fijaron en 0,90 y 0,99 . Las cargas extraordinarias se obtuvieron haciendo FDA $_{\mathrm{M}} \mathrm{e}\left(\mathrm{a}_{\mathrm{e}}\right)=0,50$ y de allí calculando el valor $\mathrm{a}_{\mathrm{e}}$.

En este trabajo se estudiaron cuatro efectos: a) Momento flector en el centro de la luz, b) Momento en un extremo, c) Carga axial de columna que soporta un piso y d) Cortante en un extremo. La constante de efecto Ke calculada para cada uno de los anteriores resultó igual a 2.80, 2.08, 2.21 y 2.21 respectivamente.

Debido a la variedad de áreas encontradas en los pisos estudiados se procedió a modelarlas como una variable denominada áreas ponderadas y conformar rangos de aplicación. La cargas unitarias de estudio $U(A)$ se calcularon dividiendo cada carga $P s$ entre el área Ac en que ésta se presentaba y luego fueron agrupadas según los rangos descritos.

Al evaluar los cambios de usuario se encontró que el tiempo promedio entre cambios es de 8,8 años resultando un valor $\mathbf{n}_{\mathrm{s}}=0,114$. Por otra parte como no se dispuso de información en cuantía y calidad suficiente sobre cargas extraordinarias fue necesario adoptar un modelo heurístico.

Del procesamiento de los datos obtenidos mediante el modelo resumido y usando el software MATLAB v.7. [3] en las ecuaciones 1 a 20 se obtuvieron los siguientes resultados:
Media de cargas sostenidas:

$$
m=0,592 \mathrm{KN} / \mathrm{m} 2
$$

Varianza de carga sostenida:

$$
\sigma_{s}^{2}=(V * 0,592)^{2}
$$

Varianza de carga según efecto:

$$
\operatorname{var}(C u(A))=10,01+116,82 * \frac{K e}{A}
$$

Varianza de carga según número de pisos y sus coeficientes de correlación:

$$
\begin{aligned}
& \operatorname{var}(C u(A=188,8 m 2))=2,05+\frac{16,04}{A} \\
& \operatorname{var}(C u(A=134,4 m 2))=3,26+\frac{6,01}{A} \\
& r_{c}=0,988 \quad y \quad r_{c}=0,92(\text { seleccionado })
\end{aligned}
$$

Parámetro que relaciona el decaimiento de la correlación:

$$
\begin{aligned}
& K(A)=0,4464+0,0472 \sqrt{\frac{A}{d}} \quad \text { con } \\
& K(A)=0,971(\text { valor medio })
\end{aligned}
$$

Usando el valor ponderado de $\sqrt{A}=6,0 \mathrm{~m}$ se obtiene $d=0,29 \mathrm{~m} 2$ lo que indica que la correlación decae hasta $\exp (-1)$ en un círculo de 0,60m de diámetro lo que ratifica la hipótesis de independencia espacial.

Los parámetros para la carga asociada a efecto carga de columnas de varios pisos resultan ser:

$$
\begin{gathered}
\sigma_{p}{ }^{2}=6,72\left(\mathrm{KN} / \mathrm{m}^{2}\right)^{2} \\
\operatorname{var}(A n)=10,01-6,72 * \frac{(n-1)}{n}+116,82 * \frac{1}{n^{*} A}+117,96 * \frac{n}{n^{*} A}
\end{gathered}
$$

Dando valores a $n$ desde 1 hasta 6 y a $A$ desde 20 hasta $640 \mathrm{~m} 2$ se ajusta la expresión:

$$
\operatorname{var}(U(A n))=6,11+\frac{252,25}{A}
$$

y usando $\mathrm{Ke}=2,21$ se obtiene para columnas:

$$
\operatorname{var}(C u(A n))=6,11+\frac{567,29}{A}
$$


Como la varianza depende de A las cargas parciales y totales pueden ser modeladas de la forma general:

$$
C t(A)=e_{1}+\frac{f_{1}}{\sqrt{A}}
$$

La tabla 1 presenta la ecuación anterior ajustada según cada efecto.

Tabla 1. Ecuaciones de ajuste para carga total según cada efecto [1], [5].

\begin{tabular}{|c|c|c|}
\hline Efecto & Percentil & Carga Total Ct(A) KN/m2 \\
\hline Momento central & 0,90 & $C t(A)=1,22+\frac{11,28}{\sqrt{A}}$ \\
\hline Momento extremo & 0,99 & $C t(A)=1,62+\frac{14,07}{\sqrt{A}}$ \\
\hline & 0,90 & $C t(A)=1,33+\frac{9,36}{\sqrt{A}}$ \\
\hline Carga de columna 1P & 0,99 & $C t(A)=1,73+\frac{11,72}{\sqrt{A}}$ \\
\hline & 0,90 & $C t(A)=1,32+\frac{9,61}{\sqrt{A}}$ \\
\hline Carga columna varios P & 0,90 & $C t(A)=1,72+\frac{12,04}{\sqrt{A}}$ \\
\hline & 0,99 & $C t(A)=1,00+\frac{12,70}{\sqrt{A}}$ \\
\hline
\end{tabular}

La práctica del análisis y diseño de estructuras exige una mayor simplificación a lo obtenido en la tabla anterior. Si se usa solo el criterio de ajuste para cada percentil de los elegidos se obtiene solo una expresión por cada uno de ellos para todos los efectos así [1], [5]:

Para percentil 0,90: $C t(A)=1,29+\frac{10,09}{\sqrt{A}}$

Para percentil 0,99: $\operatorname{Ct}(A)=1,69+\frac{12,61}{\sqrt{A}}$

FIGURA 4. Comparación de cargas totales obtenidas con la carga viva mayorada definida por la Ley 400 de 1997 (NSR-98) [1], [5].

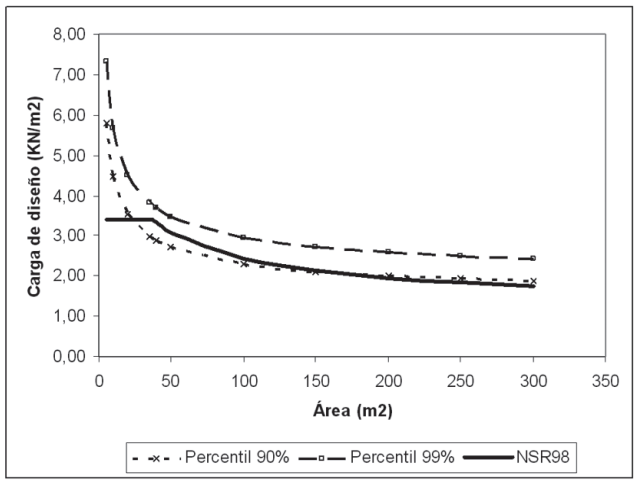

Para efectos de comparación puede obtenerse un factor de mayoración de carga viva $U$ ponderando los cocientes entre cada una de las ecuaciones anteriores con el valor esperado de la carga total que para este estudio resultó ser:

$$
E(C t(A))=1,01+\frac{8,29}{\sqrt{A}}
$$

Si se fija la carga viva mínima en $2,40 \mathrm{KN} / \mathrm{m} 2$ se obtiene:

$\mathrm{U}=1,26$ para percentil 0,90

$\mathrm{U}=1,60$ para percentil 0,99

y la ecuación B.4-1 de las NSR-98 [1] debería ser reemplazada por:

$$
L=L_{0} *\left[0,43+\frac{3,22}{\sqrt{A}}\right]
$$

\section{Conclusiones}

La aplicación de distribuciones Gamma, de Poisson y de estadígrafos apropiados es especialmente útil en la evaluación de la variabilidad de cargas en el tiempo y el espacio para estructuras de ingeniería civil.

Las expresiones para definir parámetros estadísticos simplificados de manera práctica permiten alimentar un modelo cuyos resultados pueden corroborarse con la tendencia de mediciones reales y proyectar a futuro la tendencia de la carga.

Los datos observados por Márquez y Pérez [5] permiten corroborar la variación existente de la carga de acuerdo al área de ocupación y las distribuciones de frecuencia relativa de las cargas observadas se ajustan bien a una distribución de probabilidad tipo Gamma.

En los modelos de distribución de cargas la correlación entre valores de carga de un mismo piso se puede considerar despreciable.

Según lo observado por Márquez y Pérez [5] se puede esperar que en un tiempo de vida útil de los edificios promedio de 50 años ocurran entre cinco y seis cambios de usuario en un área determinada. 
La carga mínima propuesta por las NSR-98 Márquez y Pérez [1] es de $2,00 \mathrm{KN} / \mathrm{m} 2$ y su factor de mayoración de 1,70. Ajustando los resultados reportados por Márquez y Pérez [5] a la actualidad se sugiere que la carga mínima sea de $2,40 \mathrm{KN} / \mathrm{m} 2$ y el factor de mayoración igual a 1,60 tal y como los definen las normas americanas.

Para áreas mayores que $100 \mathrm{~m} 2$ la ecuación B.4-1 de las NSR-98 [1] coincide con la carga obtenida para el percentil $90 \%$. Esto debería hacerse explícito en el texto de la Ley .

\section{Bibliografía}

Asociación Colombiana de Ingeniería Sísmica (AIS). Normas Colombianas de Diseño y Construcción Sismo Resistente. Tomos I y II. Santafé de Bogotá. 1997.

Benjamin, J., Cornell, C. Probabilidad y estadística en Ingeniería Civil. Bogotá. Mc Graw Hill. 1981. 685p. ISBN 968-451-050-0

The MathWorks. MATLAB v.7. \& SIMULINK v.6. R2007a. 2007. Software.

Corotis, R. Reability basis of live loads in standardsstate of the art. p. 86-90. En: AMERICAN SOCIETY OF CIVIL ENGINEERS. Journal of the structural division. Massachusets, 1979.

Márquez, J., Pérez, P. Determinación estadística de la variabilidad de la carga vertical viva en edificios de oficinas en la ciudad de Cúcuta. Universidad Francisco de Paula Santander. 1991. 